\title{
Radiation-Induced Dermatitis
}

National Cancer Institute

\section{Source}

National Cancer Institute. Radiation-Induced Dermatitis. NCI Thesaurus. Code C3349.

A cutaneous inflammatory reaction occurring as a result of exposure to biologically effective levels of ionizing radiation. 